\title{
La repercusión económica y jurídica del tercer principio de la Alianza Cooperativa Internacional
}

(The economic and legal impact of the third principle of the International Cooperative Alliance)

\author{
Dante Cracogna ${ }^{1}$ \\ Universidad de Buenos Aires (Argentina)
}

Sumario: I. Los principios cooperativos en la Alianza Cooperativa Internacional. II. La Declaración sobre la Identidad Cooperativa. III. El tercer principio de la Declaración sobre la Identidad Cooperativa. IV. El capital de la cooperativa y su retribución. V. El excedente cooperativo y su retribución. VI. Las reservas y el patrimonio social. VII. Conclusiones. VIII. Bibliografía.

Summary: I. The International Cooperative Alliance and the Cooperative Principles. II. The Statement on the Cooperative Identity. III. The Third Principle of the Statement on the Cooperative Identity. IV. The Cooperative Capital and its compensation. $V$. The surplus and its distribution. VI. Reserves and Equity. VII. Conclusions. VIII. Bibliography.

Resumen: El artículo trata acerca del contenido del tercer principio de la Declaración sobre la Identidad Cooperativa aprobada por la Alianza Cooperativa Internacional en 1995 tomando en consideración su proceso de elaboración y su relación con los principios incluidos en las anteriores declaraciones de esa organización aprobadas en 1937 y 1966, como así también los comprendidos en los estatutos de la Cooperativa de Rochdale, antecedentes de todas ellas. Se destaca la importancia de este principio como síntesis de los aspectos económicos fundamentales que caracterizan a las cooperativas, analizando las diferentes materias que abarca su enunciado y su relación los demás principios. Se resalta su trascendencia de cara al régimen legal de las cooperativas toda vez que los diversos temas que aborda su amplio enunciado son determinantes para caracterizar a estas entidades y delinear sus rasgos diferenciales con relación a otras formas de organización empresarial. Como conclusión se destaca la complejidad del tema y la necesidad de la adecuada investigación de sus diversas aristas.

Palabras clave: Principios cooperativos. Capital cooperativo. Interés al capital. Retornos. Reservas.

1 E-mail: dcracogna@estudiocracogna.com.ar 
Abstract: This article deals with the content of the third principle of The Statement on the Cooperative Identity approved by the International Cooperative Alliance in 1995 taking into account the process of its elaboration and the relationship with the principles included in the previous declarations of the ICA passed in 1937 and 1966 as well with those enshrined in the Rochdale Society bylaws which are the precedent of all of them. The importance of this principle as the synthesis of the basic economic aspects shaping cooperatives is well noted and the different matters involved in its text and their relations with the other principles is analyzed. Its relevance for the legal regulation of cooperatives is pointed out noting that the different aspects of its ample content are decisive in order to characterize these organizations making clear the difference with other forms of enterprises. As conclusion the complexity of the subject is pointed out as well as the need for further research of its various profiles.

Keywords: Cooperative Principles. Cooperative Capital. Interest on Capital. Patronage Refund. Reserves. 


\section{Los principios cooperativos en la Alianza Cooperativa Internacional}

La Alianza Cooperativa Internacional $(\mathrm{ACl})$ es la autoridad indiscutida en materia de doctrina de cooperativa y sus pronunciamientos son reconocidos universalmente como definitorios de la naturaleza y los caracteres propios de las cooperativas ${ }^{2}$. Ello ha venido sucediendo desde los orígenes mismos de esta organización en 1895, habiéndosele reconocido su condición de depositaria y continuadora de la tradición rochdaleana ${ }^{3}$.

El Congreso de la $\mathrm{ACl}$ realizado en Viena en 1930 consideró necesario establecer de manera precisa los principios que identifican a las cooperativas y, consiguientemente, a las entidades que calificaban para ser miembros de esa organización. A tal efecto designó una comisión especial que llevara adelante un relevamiento entre las cooperativas de diferentes actividades de los distintos países y presentara un informe en el siguiente congreso ${ }^{4}$. La comisión cumplió su cometido y presentó su informe en el Congreso realizado en Londres en 1934 pero éste consideró que debía ampliarse la encuesta a fin de que fuera cabalmente representativa del cooperativismo mundial ${ }^{5}$.

2 La propia $\mathrm{ACl}$ comienza definiendo su misión de la siguiente manera: «La $\mathrm{ACl}$ une al movimiento cooperativo mundial y es el custodio de los valores y principios cooperativos.»

3 Sin embargo, debe admitirse que existen otros enunciados de principios cooperativos formulados por diferentes organizaciones, para diversas clases de cooperativas o bien expresados por distintos autores. Una interesante referencia a distintos enunciados de principios cooperativos con repercusión jurídica puede verse en Münkner, Hans-H., Co-operative Principles and Co-operative Law, Institute for Co-operation in Developing Countries, Philipps-Universität, Marbug, 1974, passim. Por su parte, W.P. Watkins, relator de la comisión cuyo informe sirvió de base para la declaración de los principios cooperativos aprobada por el Congreso de la ACI realizado en Viena en 1966, y cuya influencia en los trabajos preparatorios de la Declaración sobre la Identidad Cooperativa de 1995 es notoria, realiza una personal enunciación de principios en su libro Co-operative Principles Today and Tomorrow, Holyoake Books, Manchester, 1986.

${ }^{4} \mathrm{ACl}$, Report of the Proceedings of the Thirteenth Congress of the International Cooperative Alliance held at Vienna 25th to 28th August 1930, The Co-operative Printing Society, London, 1931. Los integrantes de la Comisión, encabezada por Väino Tanner, constitutían un grupo muy calificado: Ernst Poisson, Albin Johansson, Víctor Serwy, J.P. Warbasse y Gromoslav Mladenatz, entre otros. Colaboró con la comisión Henry J. May, histórico secretario de la $\mathrm{ACl}$ durante muchos años.

5 ICA, Report of the Proceedings of the Fourteenth Congress of the International Cooperative Alliance held at London 4th to 7th September 1934, The Co-operative Printing Society, London, 1934. 
Finalmente, el siguiente Congreso, realizado en París en 1937, aprobó los llamados Principios de Rochdale contenidos en siete breves enunciados, A partir de entonces podían ser reputadas como cooperativas las organizaciones que, bajo cualquier régimen jurídico, observaran dichos principios ${ }^{6}$. El paso fue trascendente pues ese plexo de principios constituía un cartabón preciso para identificar a las cooperativas y, a la vez, servía de orientación para la legislación destinada a regirlas.

Años después, la misma $\mathrm{ACl}$ reconoció que el cambio operado en las condiciones económicas y sociales globales y las enseñanzas recogidas en la experiencia de la actividad cooperativa aconsejaban un ajuste en de los principios proclamados en 1937. Consiguientemente, el Congreso de Bournemouth resolvió designar una comisión que, previo encuestar a las organizaciones miembros de la $\mathrm{ACl}$ y otros, presentara un informe sobre el tema al siguiente congreso ${ }^{7}$. Así se hizo y el Congreso realizado en Viena en 1966 aprobó un nuevo enunciado de los principios cooperativos que, si bien no contiene reformas de fondo, los actualiza y formula de manera acorde a la nueva realidad mundial del momento 8 .

\section{La Declaración sobre la Identidad Cooperativa}

Transcurridas varias décadas, los cambios sobrevinientes que transformaron la fisonomía global impusieron la necesidad de una nueva adaptación que fue acogida por la $\mathrm{ACl}$ a partir del Congreso de Estocolmo (1988) y se tradujo primeramente en un debate sobre los valores cooperativos que culminó en el Congreso de Tokio de 1992 luego de un trabajo preparatorio basado en un documento del propio presidente de la ACl, Lars Marcus ${ }^{9}$, que fue motivo de amplia consulta y sis-

${ }^{6} \mathrm{ACl}$, Compte rendu du Quinzième Congrès de l'Alliance Cooperative Internationale à Paris du 6 á 9 septembre 1937, Imprimierie Nouvelle, Amiens, 1938.

$7 \mathrm{ACl}$, Report of the Twentieth Second Congress at Bournemouth 14th to 17th October 1963, The Co-operative Printing Society, London, 1964. Otra vez, la Comisión fue integrada por calificados representantes de los movimientos cooperativos de distintas regiones del mundo y de las más diversas actividades, a saber: Arnold Bonner (Gran Bretaña); Reinhold Henzler (Alemania); I.A. Kistanov (Unión Soviética); D.G. Karve (India) y Howard Cowden (EEUU). Actuó como relator W.P. Watkins, ex director de la ACl y destacado estudioso de la doctrina cooperativa.

$8 \mathrm{ACl}$, Report of the Twentieth Third Congress at Vienna 5th to 8th September 1966, The Co-operative Printing Society, London, 1967.

9 Marcus, Lars, Cooperativas y valores básicos, El Hogar Obrero, Buenos Aires, 1988. 
tematización por Sven Ake Böök asistido por un grupo internacional de expertos $^{10}$.

De allí en adelante se llevó a cabo un proceso de consulta de alcance mundial favorecido por las modernas técnicas de comunicación y llevado adelante con la participación de un numeroso equipo internacional de expertos liderado por lan Mac Pherson quien produjo un documento que sirvió de base para la discusión del tema en el Congreso del Centenario de la $\mathrm{ACl}$ realizado en Manchester en 199511. Fue un largo proceso de elaboración que concitó una notable participación y en el cual se fueron introduciendo aportes y ajustes hasta su fase final ${ }^{12}$.

Se llegó así a la aprobación de un documento que, a diferencia de los anteriormente sancionados por la $\mathrm{ACl}$, exhibe una estructura compleja compuesta de tres partes, a saber: a) inicialmente aparece, por vez primera, una definición de cooperativa que contiene los dos elementos que la doctrina venía considerando como partes inescindibles de la cooperativa: la asociación y la empresa13; b) a continuación, los valores sobre los que se basan las cooperativas y, finalmente, c) el enunciado de los siete principios mediante los cuales las cooperativas llevan a la práctica sus valores. De esta suerte, la comprensión cabal de la cooperativa exige tener en cuenta todo el complejo: definición, valores y principios, que se sustentan mutuamente ${ }^{14}$. Sin embargo, cabe señalar que, desde el punto de vista jurídico solamente resulta exigible - en la medida que la legislación respectiva los incorpore- los principios cooperativos, toda vez que la definición y los valores son, por su

10 Sven Ake Böök, Co-operative Values in a Changing World, International Co-operative Alliance, Geneva, 1992.

11 Cabe recordar que para comienzos de la década de 1990 se había producido la caída del Muro de Berlín y que el proceso de globalización económica alcanzó proporciones antes desconocidas, todo lo cual sin duda incidió en los estudios y debates acerca de la nueva formulación de los principios cooperativos.

12 Carlos Uribe Garzón efectúa una ajustada reseña de este proceso en Los principios cooperativos para el siglo XXI, Fondo Nacional Universitario, Bogotá, 1996, p. 20 y ss.

13 Fauquet, Georges, The Co-operative Sector, trad. L. Purcell Weaver, Co-operative Union Ltd., Manchester, 1951. Esta obra, originalmente publicada en francés en 1935, ha tenido gran influencia en cuanto a la afirmación del doble carácter de las cooperativas. No obstante, su carácter empresario continuó siendo resistido por muchos autores hasta que, finalmente, la ACI lo incorporó en la definición de cooperativa.

14 La Declaración Sobre la Identidad Cooperativa suele ir acompañada de un Documento de Referencia escrito por lan Mac Pherson, en el cual se explican el sentido y alcance de cada uno de sus elementos. Pero debe notarse que este documento no es parte de la Declaración y, por ende, no tiene carácter oficial sino meramente orientativo. 
naturaleza, imposibles de imponer ${ }^{15}$. A pesar de lo dicho, los principios sostienen a los otros dos elementos de la Declaración sobre la Identidad Cooperativa.

\section{El tercer principio de la Declaración sobre la Identidad Cooperativa}

Dentro de la economía de la Declaración, surge evidente que el extenso tercer principio engloba el conjunto de los aspectos económicos de las cooperativas, aun cuando los restantes principios puedan tener alguna referencia a ellos. Su propio epígrafe así lo denuncia: «Participación económica de los miembros», en tanto que los demás se refieren a ingreso voluntario, gobierno democrático, autonomía, educación, colaboración integración y preocupación por la comunidad; es decir, aspectos no específicamente económicos. De tal suerte que puede afirmarse que en este principio se concentra el perfil económico de la cooperativa y de allí su importancia, sin por ello minimizar la de los otros princi$\operatorname{pios}^{16}$. Por otro lado, debe tenerse presente, como ya lo había señalado la Comisión que produjo su informe en el Congreso de Viena (1966) que los principios se refuerzan entre sí, formando un plexo inescindible y que, por lo tanto, la cooperativa debe observar todos ellos ${ }^{17}$.

La versión española del tercer principio, si bien no oficial, reza: «Los miembros contribuyen de manera equitativa al capital de la cooperativa y lo gestionan democráticamente. Al menos una parte de dicho capital suele ser propiedad común de la cooperativa. En general los miembros reciben una compensación limitada, si la hubiera, sobre el capital aportado como requisito para pertenecer a la cooperativa. Los miembros

15 A propósito de este tema resulta de especial interés la tarea emprendida por SGECOL (Study Group on European Cooperative Law), integrado por especialistas del Derecho Cooperativo de varios países europeos (Hagen Henrÿ, Antonio Fici, Gemma Fajardo, Hans-H. Münkner, lan Snaith, David Hiez y Deolinda Meira), quienes se plantearon llevar adelante el proyecto denominado PECOL: estudio de los principios europeos del derecho cooperativo, el cual podría continuar con el de otras regiones. También son interesantes las reflexiones de Moreno Fontela, Juan Luis, "Las relaciones entre los valores y principios cooperativos y los principios de la normativa cooperativa» en REVESCO Revista de Estudios Cooperativos, n. ${ }^{\circ} 124$, Madrid, 2017, p. 114 y ss.

16 En efecto, otras entidades (asociaciones, mutuales, etc.) pueden también exhibir las características de las cooperativas definidas en los demás principios cooperativos, pero solamente las cooperativas tienen las notas que el tercer principio enuncia; por ello su particular relevancia.

17 Watkins, W.P. lo manifiesta categóricamente (Co-operative Principles Today and Tomorrow, Holyoake Books, Manchester, 1986, p. 2). 
destinan los excedentes repartibles a cualquiera de los fines siguientes: al desarrollo de la cooperativa - posiblemente mediante la creación de reservas, una parte al menos de las cuales será de carácter indivisible-, a la retribución de los miembros de manera proporcional a sus transacciones con la cooperativa y a sufragar otras actividades aprobadas por los miembros.» Independientemente de obvios problemas de redacción y de traducción, es dable advertir que en el texto se subsumen materias heterogéneas y complejas, con frases subordinadas, producto de las sucesivas versiones que se fueron adoptando hasta llegar a la definitiva.

Por otra parte, este principio conjuga temas que en las anteriores declaraciones de la $\mathrm{ACl}$ se hallaban distribuidos en más de uno, según la materia. Así, el principio de interés limitado al capital estaba separado del relativo a distribución de los excedentes. A ello debe sumarse que en el actual, aparecen cuestiones novedosas como las reservas irrepartibles, la diferencia entre aportes obligatorios y voluntarios de los miembros y la posibilidad de captar capital de fuentes externas, prevista en el cuatro principio. Todo ello exige un adecuado tratamiento para que su recepción legislativa sea consistente con la naturaleza cooperativa y para que ésta se preserve claramente diferenciada de la que corresponde a las sociedades de capital o a las asociaciones benéficas. Conforme con la definición contenida en la Declaración sobre la Identidad Cooperativa, la cooperativa es al mismo tiempo una asociación de personas y una empresa económica, por lo que no puede ser tratada exclusivamente como una u otra; y el carácter empresario se encuentra principalmente recogido en el tercer principio.

\section{El capital de las cooperativas y su retribución}

Tema inicial del principio es el relativo al capital, comenzando por afirmar que los miembros contribuyen equitativamente a su formación. De esa manera se establece claramente que todos los miembros tienen la obligación de aportar a la formación del capital de la cooperativa y que dicho aporte debe ser equitativo, es decir distribuido de manera justa entre todos ellos, sin imposiciones arbitrarias ni desigualdades discriminatorias, asegurando que todos participen con aportes acordes a las necesidades de la cooperativa pero sin exigencias que tornen limitativo el ingreso. Acerca de este tema no hacían mención las anteriores declaraciones de principios de la $\mathrm{ACl}$, posiblemente por sobreentender que el capital era aportado por los miembros puesto que la única referencia al capital estaba hecha a propósito de la limitación del interés que se podía pagar sobre él (4. ${ }^{\circ}$ principio de 1937 y 3 .er de 1966, basa- 
dos en los estatutos de Rochdale). Vale recordar que el 3. er principio de 1966 cuando se refería al capital decía específicamente: «el capital accionario», es decir el aportado por los miembros.

No obstante, a pesar de lo expresado por el principio en comentario, podría concebirse —como de hecho sucede en algunos paísesque existan cooperativas sin capital aportado por los miembros, que operan en base a la responsabilidad personal de éstos y obtienen financiamiento externo para sus actividades. En tales supuestos, obviamente no regiría la exigencia de aporte equitativo, salvo en materia de responsabilidad.

Asimismo se establece que los miembros administran el capital de manera democrática. En rigor, lo que se administra y gestiona en forma democrática es la propia cooperativa, de la cual el capital es parte. De tal suerte que la mención contenida en este principio debe entenderse como una reafirmación del gobierno democrático expresado en el 2. ${ }^{\circ}$ principio.

A continuación, el 3. er principio trata sobre la retribución o compensación del capital. Este tema, de profunda raíz rochdaleana y que constituye uno de los rasgos diferenciales más característicos de las cooperativas, estaba tratado en forma específica y diferenciada en las declaraciones de 1937 y 1966. Ahora, contenido dentro del principio «económico» general, se le introducen algunas matizaciones con relación a las anteriores enunciaciones.

En 1937 el $4{ }^{\circ}$ principio establecía, lisa y llanamente, que debía pagarse un interés limitado sobre el capital. En 1966 el 3. er principio limitó la referencia al «capital accionario» y, sin perjuicio de mantener la limitación, aclaró que podría, incluso, no pagarse interés alguno. En el actual 3. ${ }^{\text {er }}$ principio se mantiene la limitación, incluida la posibilidad de no pagar ningún interés, y se puntualiza que todo ello es con relación «al capital aportado como condición para ser miembro» de la cooperativa. Vale decir que se formula una distinción dentro del capital aportado por los miembros: el que aportan como condición para adquirir la condición de tales, conforme con la ley y el estatuto, y el que aportan por encima de esa exigencia. La limitación de la compensación solamente rige para el primero, seguramente en el entendido que de esa manera se podría estimular que los miembros aportaran capital adicional.

No se determina si el interés al capital ha de calcularse previamente a la determinación del resultado final del ejercicio económico, es decir si se considera como un gasto, o bien si debe ser extraído del excedente, es decir si es uno de los destinos del resultado final. Es verdad que si el capital es considerado un asalariado en la cooperativa, su re- 
muneración debería ser computada antes de determinar el resultado final, pero también se sostiene que el interés con que se compensa al capital debe salir del resultado, como un verdadero capital de riesgo sujeto a la suerte de la gestión económica; de otra manera sería equiparable a un préstamo, a un pasivo, y no parte del patrimonio ${ }^{18}$. Las legislaciones dan distinto tratamiento al tema, si bien en general consideran al capital de manera diferente del pasivo y, por lo tanto, el interés se detrae del resultado ${ }^{19}$.

Es del caso señalar que existen cooperativas que establecen la obligación de sus miembros de aportar capital en proporción con el uso real o potencial de los servicios; es decir que a mayor uso (real o potencial) corresponde un mayor aporte 20 . En tales casos, la compensación al capital se torna irrelevante en la medida que los miembros recibirán un retorno proporcional por sus operaciones con la cooperativa que incluirá dicha compensación.

La cuestión crítica en relación con el capital y su remuneración radica en la posibilidad de contar con aportes de miembros que se asocian sólo como «inversores», es decir sin utilizar los servicios de la cooperativa. Se trata de una novedad, inexistente en las anteriores declaraciones de la $\mathrm{ACl}$. Esta modalidad surgió a comienzos de la década de 1990 en varios países europeos (Bélgica, Italia, Francia) cuyas leyes de cooperativas autorizaron la incorporación de estos socios, lo cual provocó un notable debate doctrinario. Por una parte, se sostenía la necesidad de incorporar capital que permitiera hacer frente a las nuevas exigencias del contexto económico globalizado y, por otro, se denunciaba que con la incorporación de los llamados «socios no usuarios», o "socios inversores» se desnaturalizaba la cooperativa cuya esencia consistía en la identidad «usuario-miembro»; por lo tanto, se

18 El caso argentino es representativo: la Ley de Cooperativas dispone que «de los excedentes repartibles se destinará ... una suma indeterminada para pagar un interés a las cuotas sociales, si lo autoriza el estatuto, el cual no puede exceder en más de un punto al que cobra el Banco de la Nación Argentina en sus operaciones de descuento (art. 42, inc. $4{ }^{\circ}$ ). Por ello un prestigioso autor califica al interés como un «dividendo limitado» (Althaus, Alfredo A., Tratado de Derecho Cooperativo, 2. ${ }^{\text {a }}$ ed., Rosario, 1977, p. 47 y ss).

19 Fici, Antonio, trata este asunto en forma precisa: "An Introduction to Cooperative Law», en Dante Cracogna-Antonio Fici-Hagen Henrÿ (Editors), International Handbook of Cooperative Law, Springer, Heidelberg, 2013, p. 42-43.

20 Este sistema de capitalización proporcional es utilizado principalmente en cooperativas que requieren una importante inversión en bienes de activo fijo que se financian con los aportes de capital de sus miembros, y en tales supuestos el mayor uso de los servicios implica una mayor necesidad de capital por parte de cooperativa, lo cual justifica, a su vez, un mayor aporte de capital de los miembros. 
introducía en la cooperativa un conflicto de intereses entre los sociosusuarios y los socios-capitalistas ${ }^{21}$.

Esta discusión, intensa en el seno de la $\mathrm{ACl}$, fue transada mediante

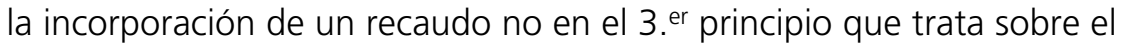
capital y su remuneración sino en el $4 .^{\circ}$ que versa sobre la autonomía e independencia de las cooperativas. De tal suerte, cuando en dicho principio se afirma que las cooperativas son organizaciones autónomas administradas por sus miembros, se dice que si entran en acuerdos con otras organizaciones, incluidos los gobiernos, "O reciben capital de fuentes externas», lo hacen en condiciones que aseguren su administración democrática por parte de sus miembros y mantengan su autonomía. De esta manera quedó resuelto el tema, admitiéndose la incorporación de capital externo —no sólo para financiación mediante crédito- pero a condición de que no atente contra la autonomía de la cooperativa ${ }^{22}$. No existe mención acerca de la compensación que puede reconocerse a este capital, pero resulta evidente que solamente será obtenible si su remuneración le resulta conveniente la cual, obviamente, superará la tasa que se abona a los miembros-usuarios comunes ${ }^{23}$.

Huelga decir que - a pesar del equilibrio que se trató de mantener mediante este principio- la aceptación de tal modalidad de incorporación de capital continúa siendo materia de controversia ${ }^{24}$. Es obvio señalar que resulta por completo diferente la asunción de otras formas de pasivo por parte de las cooperativas para su financiación, tales como empréstitos, bonos u otras modalidades que no impliquen participación en las decisiones sociales; en este caso no existen observaciones, sólo debe cuidarse la adecuada gestión del endeudamiento ${ }^{25}$.

21 Sobre los riesgos que entraña la admisión de capital externo alerta Henrÿ, Hagen, Guidelines for Cooperative Legislation, third revised edition, International Labour Office, Geneva, 2012, p.12.

22 Es lo que sucede en la legislación italiana que admite que los socios capitalistas participen en los órganos de la cooperativa con no más del treinta por ciento de sus integrantes.

23 Un interesante relevamiento, con consideraciones favorables a la incorporación de inversores externos, puede consultarse en Andrews, A. Michel, Survey of Co-operative Capital, International Co-operative Alliance, Filene Research Institute, Madison, 2015.

${ }^{24}$ Cfr. Kaplan de Drimer, Alicia, «Enemigos invisibles: el caso de la CWS de Gran Bretaña», Anuario de Estudios Cooperativos, Universidad de Deusto, Bilbao, 2000.

25 La Ley Marco para las Cooperativas de América Latina admite expresamente que las cooperativas asuman toda clase de pasivos (art. 45) pero en cuanto a capital de fuentes externas solamente lo permite cuando proviene de cooperativas de cualquier grado, entidades sin ánimo de lucro, agencias nacionales e internacionales de fomento 
La trascendencia actual del tema del capital en las cooperativas queda evidenciada por cuanto el Plan para una Década Cooperativa aprobado por la asamblea extraordinaria de la $\mathrm{ACl}$ realizada en Manchester en 2012 cuando se clausuraba el Año Internacional de las Cooperativas declarado por las Naciones Unidas lo incluye entre sus cinco ejes fundamentales. Señala al respecto el documento: "Obtener capital fiable para las cooperativas al mismo tiempo que se garantice el control por parte de los socios» ${ }^{26}$, con lo cual evidencia que considera viable el recurso a inversores externos, sin bien con recaudos. Esto último se halla explícitamente reconocido cuando el aludido documento se refiere a la necesidad de contar con marcos legales que apoyen el desarrollo cooperativo y previene acerca de la reproducción de prácticas de las empresas de propiedad de inversores, con lo cual las cooperativas se arriesgan a perder su identidad ${ }^{27}$.

Por otra parte, debe tenerse presente el problema que ha provocado a las cooperativas, especialmente las financieras, la consideración del capital aportado por sus miembros como parte del pasivo y no como integrante del patrimonio, en razón de que puede ser devuelto cuando aquéllos se retiran. Esta caracterización del capital cooperativo por las NIIF (Normas Internacionales de Información Financiera) es una muestra de las graves consecuencias que el soft law producido por fuentes no legislativas - en este caso las organizaciones de la profesión contable- es capaz de provocar ${ }^{28}$.

\section{El excedente cooperativo y su distribución}

Otro tema de singular relevancia que trata el $3 .^{\text {er }}$ principio es el relativo a la distribución de los excedentes. Quizás sea éste, junto con el de la democracia, el principio más definitorio del carácter peculiar

\footnotetext{
y el Estado, en todos los casos con la limitación de participación en los órganos sociales similar a la establecida por la ley italiana, es decir no superior a la tercera parte. Sobre este aspecto y, en general, sobre el capital y el financiamiento en la Declaración de 1995 cfr. Cracogna, Dante, «Aproximación al problema del capital y el financiamiento cooperativo», en Cuadernos de Economía Social, Instituto Argentino de Investigaciones de Economía Social (IAIES), N. ${ }^{\circ}$ VIII-12, Buenos Aires, 2001, p.91 y ss.

$26 \mathrm{ACl}$, Plan para una Década Cooperativa, s/d, 2013, p. 32.

$27 \mathrm{ACl}$, Plan para una Década Cooperativa, s/d, 2013, p. 26

28 Sobre este complicado asunto, ver: Cracogna, Dante, «El capital en las cooperativas y las NIIF», en Derecho Contable Aplicado, Eduardo M. Favier Dubois, Director, Instituto Autónomo de Derecho Contable (IADECO), Errepar, Buenos Aires, 2012, p. 369 y ss.
} 
de las cooperativas. En efecto, desde Rochdale el llamado principio de Howarth ha sido considerado como piedra angular de la caracterización económica de las cooperativas: la devolución del excedente a quienes lo produjeron; es decir «el retorno» en proporción al uso de los servicios como rasgo propio y diferenciador de las cooperativas 29 . Sin embargo, este principio ya había quedado mediatizado en 1966, siguiendo la impronta común de los países socialistas, dando pie a las modernas orientaciones de las llamadas cooperativas sociales y de la economía social en general en detrimento de la promoción de los miembros ${ }^{30}$.

Se prevén cuatro opciones como destinos de la distribución del excedente: a) desarrollo de la cooperativa; b) constitución de reservas; c) distribución entre los miembros en proporción a sus operaciones con la cooperativa; y d) apoyo a otras actividades aprobadas por los miembros. Según dispone el principio, el destino, cualquiera sea, debe ser decidido por los miembros, quienes pueden resolver destinar todo el excedente a uno de los indicados o dividirlo entre dos o más de ellos.

La declaración de 1937 (4. ${ }^{\circ}$ principio), siguiendo la línea de la Cooperativa de Rochdale, establecía que los excedentes se distribuían en proporción a las operaciones realizadas por los miembros con la cooperativa. Pero la declaración de 1966, sin eliminar esa posibilidad, introdujo una significativa variante. En primer lugar afirmó que los excedentes son los que provienen de las operaciones de la cooperativa; es decir, de la actividad propia de su objeto social y, aunque no lo diga expresamente, debe entenderse que es la actividad realizada con sus miembros; por ello seguidamente sostiene que tales excedentes pertenecen a los miembros y que deben distribuirse de manera que se evite que un miembro obtenga ganancias a expensas de otro. Hechas estas

29 Holyoake, célebre historiador de la Cooperativa de Rochdale y prácticamente contemporáneo de los pioneros destaca que una clave del éxito de esa cooperativa fue la adopción de este sistema de distribución de excedentes impulsado por Charles Howarth, si bien ya en 1822 lo había anticipado Alexander Campbell en Glasgow (Holyoake, Georges J., Historia de los pioneros de Rochdale, trad. Bernardo Delom, Intercoop, Buenos Aires, 2016, p. 23 y ss). Una interesante disquisición acerca de este principio, sus antecedentes y su incorporación en el estatuto de Rochdale se encuentra en Balay, Esteban, Bases del ordenamiento cooperativo de la Economía Social, Chiesino, Buenos Aires, 1965, p. 199 y ss.

${ }^{30} \mathrm{Cfr}$. las reflexiones de Munkner, Hans-H., Co-operative Principles and Co-operative Law, Friedrich-Ebert-Stiftung, Institute for Co-operation in Developing Countries, Philipps-Universität, Marburg, 1974, p. 11 y ss. Para las cooperativas sociales resulta ilustrativo consultar la legislación y la experiencia italianas que fueron precursoras en esta materia (Cfr. Fici, Antonio, Impresse cooperative e sociali. Evoluzione normativa, profili sistematici e questioni applicative, G. Giappichelli Editore, Torino, 2012, passim). 
precisiones de orden conceptual, a continuación habilita tres destinos, a decidir siempre por los miembros: a) desarrollo de las actividades de la cooperativa; b) servicios comunes y c) distribución entre los miembros a prorrata de sus operaciones con la cooperativa. Como es dable advertir, la declaración de 1995 no contiene mayores diferencias con la de 1966 pues, aparte de la reiteración de la distribución en proporción a las operaciones, los restantes destinos son prácticamente equiparables.

Lo que ha quedado claro en ambas declaraciones es que ya no resulta imperativo el principio del retorno, que desde el punto de vista económico constituye el núcleo que diferencia a las cooperativas de las empresas de capital $^{31}$ y se ha producido una notable aproximación con las asociaciones sin fines de lucro y las mutuales, que destinan sus resultados a mayores servicios o actividades de interés general. En suma, ahora el excedente que generan los miembros con su actividad $-\mathrm{y}$ que les pertenece- pueden ellos destinarlo a desarrollo de la cooperativa o a fines de interés común. Este último destino puede considerarse en línea con el $7 .^{\circ}$ principio que establece la preocupación por la comunidad pero debe tenerse en cuenta que los excedentes constituyen una fuente primordial de la capitalización de las cooperativas, sea por vía de la constitución de reservas o - principalmente- del pago del retorno mediante nuevos aportes de capital de los miembros. Al eliminarse el retorno desaparece esta fundamental fuente de capitalización.

\section{Las reservas y el patrimonio social}

Un tercer aspecto relevante que trata el 3 er principio es el relativo a las reservas indivisibles, tema que no había sido incluido de manera expresa en las anteriores declaraciones de la $\mathrm{ACl}$. En dos aspectos se hace referencia al tema: en primer lugar, mencionando que una parte al menos del capital debe ser propiedad común de la cooperativa y, en segundo término, estableciendo que el destino de los excedentes puede ser la constitución de reservas, de las cuales al menos una parte debe ser indivisible.

El tema de la indivisibilidad de las reservas tiene antigua presencia en la doctrina y la práctica de las cooperativas. En la reforma del estatuto de la Cooperativa de Rochdale realizada en 1854 se incluyó expresamente que el sobrante del activo en caso de liquidación debía ser

31 El sentido del retorno se halla explicado en Cracogna, Dante, Comentarios a la Ley de Cooperativas, 3. ${ }^{a}$ edición actualizada, Intercoop, Buenos Aires, 2006, p. 54-56. 
destinado a fines caritativos o públicos ${ }^{32}$. A su vez, pese a que las declaraciones de 1937 y 1966 no contienen referencias a este asunto, los informes que las respectivas comisiones pusieron a consideración de ambos congresos incluyen específicas referencias a las reservas inalienables y la indivisibilidad de los fondos colectivos ${ }^{33}$, lo que demuestra la importancia que se le asignaba.

Cuando se discutía el contenido de la Declaración sobre la Identidad Cooperativa, el movimiento cooperativo francés, especialmente, adoptó una firme posición en punto a la adopción de la indivisibilidad de las reservas, la cual fue finalmente incorporada aunque sin excesivo rigor, más bien como recomendación ${ }^{34}$. Es evidente que se trató del resultado de una suerte de negociación que se tradujo, por último, en el uso de expresiones tales como «por lo general» y «posiblemente».

Además de su obvio significado doctrinal como afirmación de la propiedad común de la cooperativa, más allá de las personas y del tiempo, las reservas indivisibles tienen evidentes efectos prácticos. Conforman una parte del patrimonio que está a cubierto de la variabilidad del capital que es característica en las cooperativas, permaneciendo en forma definitiva, y son una fuente de financiación sin costo. Por otra parte evitan la tentación de disolver y liquidar cooperativas que gozan de buena situación para apropiarse de las reservas, como ha sucedido con frecuencia en los casos llamados de «desmutualización» que tuvieron lugar principalmente entre fines del siglo pasado y comienzos del presente. Esos casos, además entrañan una manifiesta injusticia toda vez que los miembros de la cooperativa que se liquida se apropian de reservas formadas, quizás, a lo largo de varias generaciones ${ }^{35}$.

32 Lambert, Paul, La Doctrine Coopérative, Les Propagateurs de la Coopération, Bruxelles, La Fédération Nationale des Coopératives de Consommation, Paris, 1959, p. 309-310. Esta obra reproduce como anexo el texto de los estatutos de la Cooperativa de Rochdale en su versión original de 1844 y la reforma de 1854, señalando las dificultades habidas para obtener tales documentos. Por otra parte, se recuerda que el 3. ${ }^{\circ}$ Congreso Cooperativo celebrado en Londres en 1832 bajo la presidencia de Robert Owen había incorporado, por unanimidad, el capital indivisible como parte del reglamento para las cooperativas ( $\mathrm{ACl}$, Notas de orientación para los principios cooperativos, s/d, 2015, p. 31).

$33 \mathrm{ACl}$, Compte rendu du Quinzième Congrès de l'Alliance Coopérative Internationale à Paris, du 6 a 9 septembre 1937, Imprimierie Nouvelle, Amiens, 1938, p. 182-183; $\mathrm{ACl}$, Nuevos enfoques de los principios cooperativos en el mundo, Idelcoop, Rosario, 1987 p. 60 y ss (traducción del informe de la comisión presentado en el Congreso de Viena, 1966).

$34 \mathrm{ACl}$, Notas de orientación para los principios cooperativos, s/d, 2015, p. 31-33.

35 Estas desviaciones están claramente denunciadas por Uribe Garzón, Carlos, Bases del cooperativismo, Fondo Nacional Universitario, Bogotá, 2002, p. 267. 


\section{Conclusiones}

La repercusión del tercer principio, tanto en el campo económico como en el jurídico, resulta de innegable trascendencia toda vez que aquél encierra las notas tipificantes de las cooperativas desde el punto de vista económico las que, a su turno, han de ser recogidas en su régimen jurídico. Sin embargo, como se señala a lo largo del trabajo, existen cuestiones que no se hallan claramente definidas o que admiten variantes, con lo cual las conclusiones —especialmente en el terreno legislativo- quedan sujetas a criterios interpretativos, susceptibles de conclusiones distintas. El interrogante que permanece es si tales cuestiones podrían ser objeto de una definición categórica o si, por su naturaleza y las circunstancias del entorno económico y social actual, deben necesariamente quedar abiertas a diversas posibilidades sin que ello signifique desvirtuar la esencia cooperativa ${ }^{36}$.

\section{Bibliografía}

ACl. 1931. Report of the Proceedings of the Thirteenth Congress of the International Cooperative Alliance held at Vienna 25th to 28th August 1930, The Co-operative Printing Society, London.

ICA. 1934. Report of the Proceedings of the Fourteenth Congress of the International Cooperative Alliance held at London 4th to 7th September 1934, The Co-operative Printing Society, London.

$\mathrm{ACl}$. 1938. Compte rendu du Quinzième Congrès de l'Alliance Cooperative Internationale à Paris du 6 á 9 septembre 1937, Imprimierie Nouvelle, Amiens.

ACl. 1964. Report of the Twentieth Second Congress at Bournemouth 14th to 17th October 1963, The Co-operative Printing Society, London.

ACI. 1967. Report of the Twentieth Third Congress at Viena 5th to 8th September 1966, The Co-operative Printing Society, London.

$\mathrm{ACl}$. 1987. Nuevos enfoques de los principios cooperativos en el mundo, Idelcoop, Rosario.

ACI. 1996. Los principios cooperativos para el siglo XXI, Intercoop, Buenos Aires.

ACl. 2013. Plan para una Década Cooperativa, s/d.

ACl. 2015. Notas de orientación para los principios cooperativos, s/d.

$\mathrm{ACl}$. Américas. 2009. Ley Marco para las Cooperativas de América Latina, San José.

36 Las Notas de orientación para los principios cooperativos elaboradas por un grupo de expertos convocados por la $\mathrm{ACl}$, concebidas para aclarar los alcances de los principios, no logran superar algunas dudas e interrogantes en esta materia. 
ANDREWS, A. Michel. 2015. Survey of Co-operative Capital, International Cooperative Alliance, Filene Research Institute, Madison.

BALAY, Esteban. 1965. Bases del ordenamiento cooperativo de la Economía Social, Chiesino, Buenos Aires.

BÖÖK, Sven Ake. 1992. Co-operative Values in a Changing World, International Co-operative Alliance, Geneva.

CRACOGNA, Dante. 2001. «Aproximación al problema del capital y el financiamiento cooperativo», en Cuadernos de Economía Social, Instituto Argentino de Investigaciones de Economía Social (IAIES), n. ${ }^{\circ}$ VIII-12, Buenos Aires.

CRACOGNA, Dante. 2006. Comentarios a la ley de cooperativas, 3a. edición actualizada, Intercoop, Buenos Aires.

CRACOGNA, Dante. 2009. «La distribución de los excedentes en las cooperativas. Derecho y contabilidad», en Doctrina Societaria y Concursal, n. ${ }^{\circ} 265$, Buenos Aires, p. 1307 y ss.

CRACOGNA, Dante. 2012. "El capital en las cooperativas y las NIIF», en Eduardo M. Favier Dubois (Director), Derecho Contable Aplicado, Instituto Autónomo de Derecho Contable (IADECO), Errepar, Buenos Aires.

DRIMER, Bernardo y Kaplan de Drimer, Alicia. 2017. Las cooperativas. Fundamentos. Doctrina. Historia, 4. ${ }^{a}$ ed. actualizada por Mirta Vuotto, Intercoop, Buenos Aires.

FAUQUET, Georges. 1951. The Co-operative Sector, trad. L. Purcell Weaver, Co-operative Union Ltd., Manchester.

$\mathrm{FICl}$, Antonio. 2013. "An Introduction to Cooperative Law», en Dante Cracogna-Antonio Fici-Hagen Henrÿ (Editors), International Handbook of Cooperative Law, Springer, Heidelberg.

$\mathrm{FICl}$, Antonio. 2012. Impresse cooperative e sociali. Evoluzione normativa, profili sistematici e questioni applicative, G. Giappichelli Editore, Torinio.

HENRŸ, Hagen. 2012. Guidelines for Cooperative Legislation, third revised edition, International Labour Office, Geneva.

HOLYOAKE, Georges Jacob. 2016. Historia de los pioneros de Rochdale, trad. Bernardo Delom, Intercoop, Buenos Aires.

LAMBERT, Paul. 1959. La doctrine coopérative, Les Propagateurs de la Coopération, Bruxelles, La Fédération Nationale des Coopératives de Consommation, Paris.

MORENO FONTELA, Juan Luis. 2017. «Las relaciones entre los valores y principios cooperativos y los principios de la normativa cooperativa» en REVESCO Revista de Estudios Cooperativos, n. ${ }^{\circ} 124$, Madrid, p. 114 y ss.

MÜNKNER, Hans-H. 1974. Co-operative Principles and Co-operative Law, Institute for Co-operation in Developing Countries, Philipps-Universität, Marbug (Second revised edition, LIT Verlag, Zurich, 2015).

URIBE GARZÓN, Carlos. 2002. Bases del cooperativismo, Fondo Nacional Universitario, Bogotá.

URIBE GARZÓN, Carlos. 1996. Los principios cooperativos para el siglo XXI, Fondo Nacional Universitario, Bogotá.

WATKINS, William P. 1986. Cooperative principles today and tomorrow, Holyoake Books, Manchester. 


\section{Derechos de autor}

El Boletín de la Asociación Internacional de Derecho Cooperativo es una revista de acceso abierto lo que significa que es de libre acceso en su integridad inmediatamente después de la publicación de cada número. Se permite su lectura, la búsqueda, descarga, distribución y reutilización legal en cualquier tipo de soporte sólo para fines no comerciales y según lo previsto por la ley; sin la previa autorización de la Editorial (Universidad de Deusto) o el autor, siempre que la obra original sea debidamente citada (número, año, páginas y DOI si procede) y cualquier cambio en el original esté claramente indicado.

\section{Copyright}

The International Association of Cooperative Law Journal is an Open Access journal which means that it is free for full and immediate access, reading, search, download, distribution, and lawful reuse in any medium only for non-commercial purposes, without prior permission from the Publisher or the author; provided the original work is properly cited and any changes to the original are clearly indicated. 